\title{
The psychometric properties of an Arabic version of the PedsQL Multidimensional Fatigue Scale tested for children with cancer
}

\begin{abstract}
Fatigue is considered to be one of the most reported symptoms experienced by children with cancer. A major aim of this study was to develop an Arabic version of the Paediatric Quality of Life (PedsQL) Multidimensional Fatigue Scale (child report) and to test its psychometric proprieties for the assessment of fatigue in Arabic children with cancer. The PedsQL Multidimensional Fatigue Scale (Arabic version) and the PedsQL TM 4.0 Generic Core scale (existing Arabic version) were completed by 70 Jordanian children with cancer. Cronbach's alpha coefficient was found to be 0.90 for the total PedsQL Multidimensional Fatigue Scale (Arabic version), 0.94 for the general fatigue sub-scale, 0.67 for the sleep/rest fatigue sub-scale and 0.87 for the cognitive fatigue sub-scale. The PedsQL Multidimensional Fatigue Scale scores correlated significantly with the PedsQL TM 4.0 Generic Core scale and demonstrated good construct validity. The results demonstrate excellent reliability and good validity of the PedsQL Multidimensional Fatigue Scale (Arabic version) for children with cancer. This is the first validated scale that assesses fatigue in Arabic children with cancer. The English scale has been used with several paediatric clinical populations, so this Arabic version may be equally useful beyond the field of cancer.
\end{abstract}

Key words: Arabic translation; cancer; children; Fatigue; Jordan; instrument-development. 


\section{Introduction}

Fatigue is one of the most common reported symptoms among children with cancer (Campos, Hassan, Riechelmann \& Del Giglio, 2011; Tomlinson, Hinds, Ethier, Ness, Zupanec \& Sung, 2013). Fatigue in children has been identified as a subjective, multidimensional concept that encompasses physical, emotional, and cognitive tiredness that could interfere with the activity daily living (Gibson, Mulhall, Richardson, Edwards, Ream, \& Sepion, 2005; Tomlinson et al 2013), quality of life (Varni, Burwinkle, Katz, Meeske, \& Dickinson, 2002a; Kobayashi, Okano \& Hohashi, 2011) and school attendance (Bakker, van de Putte, Kuis, \& Sinnema, 2009). Screening and treatment of fatigue in children with cancer may therefore lead to significant improvement in quality of life and improve the effectiveness of therapeutic interventions.

In 2010 in Jordan, 231 children between 1 to 14 years were diagnosed with a new cancer (Ministry of Health 2010). Despite this high incidence in children, there has been no reliable and valid scale available to measure fatigue in such patients. To use a reliable and valid instrument to assess the clinical level of fatigue in Arabic children with cancer was considered to be of vital importance. The most commonly used instruments to measure fatigue among children were the Paediatric Functional Assessment of Chronic Illness Therapy-Fatigue (Lai et al. 2007), the Fatigue Scale-Child/Fatigue Scale-Adolescent (Hockenberry et al., 2003; Hinds et al., 2007), PedsQL Multidimensional Fatigue Scale (Varni et al, 2002a), Memorial Symptom Assessment Scale (Portenoy, et al., 1994), Daily Fatigue Report Scale (Erickson et al, 2010) and McCorkle Symptom Distress Scale (McCorkle, 1987). The PedsQL Multidimensional Fatigue Scale is one of the most widely used child-report questionnaires for assessing fatigue in children with cancer. It is shown to be valid and reliable for the measurement of fatigue among children with different health conditions (Tomlinson et al 2013). 
The authors of this study used The PedsQL Multidimensional Fatigue Scale because it is a unique instrument since it measures the multidimensional aspect of fatigue: general fatigue (6 items), sleep/rest fatigue (6 items) and cognitive fatigue (6 items) (Varni et al, 2002a). The PedsQL Multidimensional Fatigue Scale was designed as a generic symptom-specific instrument to measure fatigue in pediatric patients aged 2-18 years from the perspective of the children, adolescents, and their parents. The author intended to check the psychometric prosperities of the Arabic version with children with cancer, since it has been reported as being a short, simple questionnaire for assessment of the fatigue and in the light of its empirical derivation and extensive validation processes (Varni, Burwinkle, \& Szer, 2004; Varni \& Limbers, 2008; Tomlinson et al 2013 ). A valid and reliable tool can help clinicians and researchers to assess fatigue in diagnostic groups in order to provide appropriate screening and interventions. The PedsQL Multidimensional Fatigue Scale was developed originally in English (Varni et al., 2002a). It has been translated to many languages such as Portuguese (Brazil) (Nascimento et al., 2015), Japanese (Kobayashi et al, 2011), and Dutch (Gordijn, Cremers, Kaspers, \& Gemke, 2011). To the authors' knowledge, the psychometric properties of the Arabic version of the PedsQL Multidimensional Fatigue Scale have not been established in children with cancer. The aim of this work was to develop the Arabic version of The PedsQL Multidimensional Fatigue Scale and test its reliability and validity for the assessment of fatigue in Arabic children with cancer in Jordan. Arabic is the first spoken language of Jordan.

The work to test the psychometric properties of this instrument was undertaken as part of a study to establish the links between self-esteem, fatigue and health-related quality of life in children with a diagnosis of cancer (The Authors 2016). 


\section{Method}

\section{Design}

A cross-sectional design was used in this study. Data were collected in 2015 as part of the main study.

\section{Participants}

The children ranged in age from 5 years to 18 years (mean=10.17 years, $\mathrm{SD}=3.4$ years). There were 30 girls and 40 boys. They had been diagnosed with range of childhood cancer for example: the majority of children $(34,46 \%)$ had leukemia, four $(5.7 \%)$ had lymphoma, and $32(48.3 \%)$ had other types of cancer. The most recent statistics from the Jordan Cancer Registry (for the year 2010) at the time of recruitment indicated that the distribution of new cases of cancer in children aged 0-14 years by sex was male $56.7 \%$ and female $43.3 \%$. The top five cancer types in this age group were leukaemia $38 \%$, brain and CNS $27 \%$, lymphoma $20 \%$, kidney $8 \%$ and soft tissue cancers $7 \%$. The sample in this study, while not intended to be directly representative, was not distinctly skewed from the national profile.

The inclusion criteria were that the child was aged 5-18 years and had been diagnosed with a cancer (of any sort), that the child could speak Arabic, and that they were well enough to participate in the study. Children in a terminal phase of illness were excluded due to the high level of fatigue which could bias the result, and which could also cause an extra burden on them. With the exception of four patients attending follow-up clinic, all case were in active treatment.

\section{Measures}

Instruments

- The Demographic Questionnaire 
For the purpose of this study the researchers developed a questionnaire to collect demographic information about the children which included age at diagnosis, sex, cancer type, and socioeconomic background.

- The PedsQL Generic 4.0 Module scales

Arabiat, Elliott, Draper \& Al Jabery (2011) have established the validity and reliability of the Arabic version of this instrument through a study in Jordan, with reported Alpha of 0.90 . This scale measures four domains of physical, emotional, social and school functioning (Varni, Seid, Knight, Uzark, \& Sze, 2002b). Permission to use the Arabic (Jordan) validated version of this instrument was granted by Mapi Trust (the owner of the PedsQL suite: https://eprovide.mapi-trust.org/).

- The PedsQL Multidimensional Fatigue Scale

This scale addresses general, sleep and cognitive-related aspects of fatigue through an 18-item questionnaire (Varni et al, 2002a). The reliability of the original scale was 0.89 . The validity of the scale has been confirmed through correlation between generic and cancer-specific health-related quality of life (Varni et al 2002a). There are three child self-report versions depending on the age of the child: 5-7 years (young child), 8-12 years (child) and 13-18 years (adolescent). A five point Likert scale is adopted for ages $8-18$ years $(0=$ never a problem; $1=$ almost never a problem, $2=$ sometimes a problem, $3=$ often a problem, 4=almost always a problem). For younger children (5-7 years), the Likert scale is simplified to a 3 -point scale $(0=$ not at all a problem; $2=$ sometimes a problem; $4=a$ lot of a problem). Higher scores indicate fewer or less intense fatigue symptoms. Items are reversed-scored and linearly transformed to a $0-100$ scale $(0=100,1=75,2=50,3=25,4=0)$. 
The main researcher gained permission from Mapi Trust to use a non-verified Arabic version of this instrument. Brislin's (1986) widely-used model of back-translation was adopted to ensure that the translated instrument bore fidelity in meaning to the original. Detail of this is provided in Al-Gamal et al (2009), but, in brief, the original version was translated by a bi-lingual academic into Arabic, this version was translated back to English by a second linguist, and the final version compared to the original English version. Discussion of finer points addressed any minor discrepancies. Experts in the field were asked to evaluate the validity of the instrument, considering individual items as well as the entire instrument for relevance, appropriateness and coverage of essential issues. The revised questionnaire was found to be unambiguous and each item was thought to be appropriate and applicable to children with cancer. All items of the revised scale were retained for further psychometric testing.

Ten Jordanian children undertook a pilot test of the Arabic version as a final check. This led to further minor modification of terminology to improve understanding by children: all matters of minimum changes to words to improve Arabic idiom rather than alteration of meaning. The average time for completion was 10 minutes. The researchers then felt confident to move to psychometric testing without further amendment to the instrument, sothe translated version, together with The PedsQL Generic 4.0 Module Scale was administered to a sample of children with cancer.

\section{Ethical approval and ethical considerations}

Research ethics approval was obtained from the Research Ethics Committee at the Faculty of Nursing, the University of Jordan and from the targeted healthcare institution. Data collection was conducted in a private room in an out-patient clinic area at the hospital in Jordan which provides the bulk of oncology care for the country. Senior nurses helped to identify eligible children with an 
appointment in the clinic, and they also undertook an initial introduction to the study. Signed consent was requested from the parents and children for patients aged 13-18 years old. For younger children (512) years, verbal assent was accepted together with written consent of their parents agreeing to allow their children to participate in the study. Research assistants were available to assist young children by reading the items and possible responses for the child to select.. Children who were able to do so, and particularly those aged 13-18 years, completed the questionnaires without assistance, though with a researcher present in case of need.

The voluntary nature of participation was emphasized verbally and in writing. To maintain privacy, no names or contact details were attached to the questionnaires and physical privacy was maintained through the exclusion of others while the questionnaires were being completed. Each child had an identification number. The participants were assured that the information provided would be used only for the stated purpose of the study, and that the decision to participate or not would not affect the child's treatment or relationship with healthcare providers.

\section{Data analysis}

Data analyses were carried out using IBM, SPSS 21 for Windows. Descriptive statistical analysis (frequency count, percentage, mean, and standard deviation) was applied to describe the sample and to summarise the PedsQL Multidimensional Fatigue Scale scores. To assess internal consistency, itemscale correlations (using Pearson correlation coefficient) and the calculation of Cronbach's alpha were used. This was calculated for the total PedsQL Multidimensional Fatigue Scale as well as for each subscale. Cronbach's alpha coefficient and alpha equal to or greater than 0.70 was considered satisfactory (Cronbach, 1951). Individual items of the PedsQL Multidimensional Fatigue Scale were reviewed for their effect on overall internal consistency. Convergent construct validity with the PedsQL 
Generic 4.0 Module scales was analyzed using Pearson's product-moment correlation, and r equal to 0.40 or above was considered satisfactory (Polit \& Beck, 2008). Statistical significance was set at $\mathrm{p}=<0.05$.

\section{Results}

\section{Degrees of fatigue}

The mean PedsQL Multidimensional Fatigue total score was 65.79 ( $\mathrm{SD}=22.20)$, while the mean scores for general fatigue, sleep/rest fatigue and cognitive fatigue were (65.79 (SD=33.1), 60.86 (SD $=24.6)$ and $70.90(\mathrm{SD}=26.15)$, respectively.

\section{Internal consistency and reliability}

Item-total scale correlations ranged from 0.41 to 0.77 , item-general fatigue subscale correlations (range 0.82- 0.91), item-sleep/rest subscale correlations (range 0.43-0.75), and item-cognitive fatigue subscale correlations (range $0.53-0.90)$ were calculated. All were statistically significant $(\mathrm{p}<0.01)$, and are shown in Table 1.

\section{[Insert Table 1 here]}

A Pearson Product Moment Correlation Matrix determined correlations between the subscales and total PedsQL Multidimensional Fatigue Scale score. Statistically significant correlations were found between total PedsQL Multidimensional Fatigue Scale score and general fatigue subscale, sleep/rest subscale and cognitive fatigue subscale at $0.859,0.709$, and .784 respectively. Cronbach's alpha for total scale, general fatigue subscale, sleep/rest subscale and cognitive fatigue subscale were 0.90, 0.94, 0.67 and 0.87 respectively. See Table 2.

\section{[Insert Table 2 here]}


The effect of individual items on the reliability of their composite subscale was tested using the Alpha if item deleted approach. Cronbach alpha was calculated when each of the items in its composite subscale was removed from the subscale. Removing any individual item from the general fatigue subscale resulted in minimal changes in Cronbach's alpha, ranging from 0.925 to 0.938 , indicating that all six items in the general fatigue subscale should be retained. This was also the case for the sleep/rest subscale ( 0.564 to 0.686$)$, and the cognitive fatigue subscale $(0.827$ to 0.910$)$, indicating that the 6 items in each of these subscales should also be retained.

\section{Construct validity}

In addition to content validity, construct validity was tested in this study. PedsQL Multidimensional Fatigue Scale total scores demonstrated significant correlations with the PedsQL Generic 4.0 Module scales (Table 3). PedsQL Multidimensional Fatigue total components were significantly associated with all subscales of the PedsQL Generic 4.0 Module scales (Table 3). The positive correlation coefficients indicated that higher Multidimensional Fatigue Total Scale and Subscale scores (fewer problems or symptoms) were correlated with higher Generic Core Total Scale Scores (better overall HRQOL).

[Insert Table 3 here]

\section{Discussion}

We report, as part of a study of the links between fatigue and other factors in children with cancer, efforts to develop an Arabic version of the PedsQL Multidimensional Scale and to test its reliability and validity for the assessment of fatigue in children with cancer in Jordan. Fatigue has been reported to be one of the most troublesome symptoms for children and adolescents undergoing treatment for 
cancer (Gibson et al., 2005; Campos, Hassan, Riechelmann, \& Del Giglio, 2011). Parents in a Turkish study of fatigue in children with cancer rated fatigue higher than the children or nurses, though nurses' scores tended to agree with children's scores (Y1lmaz, Gerçeker \& Muslu, 2016). Most PedsQL instruments, in addition to offering versions for a number of age groups, also provide for a proxy response by a parent if the child is unable to respond directly. The Turkish study did not use the PedsQL instrument, but it still offers a timely reminder of the potential for conflicting scores by proxies. Importantly, differences in fatigue were found on successive days, indicating the need for routine daily review of this common aspect of children's cancer experience.

Addressing fatigue in childhood cancer patients is of vital importance, not only during treatment and immediate follow-up, but also into adulthood. A Norwegian population-based study discovered a high prevalence of chronic fatigue in such survivors (Jóhannsdóttir et al., 2012). This indicates that nurses and other health care providers should conduct thorough assessments of the symptom burden in this patient population, screening particularly for fatigue, and intervening accordingly.

The lack of a rigorous measure for the assessment of fatigue in Arabic children may limit nursing assessment and intervention relating to fatigue in this population. A recent Brazilian study with 53 varied health professionals found evidence of lack of knowledge about fatigue, its identification, and appropriate interventions in children and adolescents with cancer (Miyauti-Silva, Lopes-Júnior, Nascimento, \& Lima, RAG, 2016). Clearly, a structured means to assess fatigue must be part of the strategy to overcome these deficits, clarifying the nature of fatigue, aiding identification, and perhaps guiding the choice of targets for intervention. 
Findings from a qualitative study in the USA (Davies, Whitsett, Bruce \& McCarthy, 2002) indicated that children with cancer report three discrete presentations of fatigue according to varying levels of energy: typical tiredness, treatment fatigue, and shutdown fatigue. Children adopted varied strategies to manage their lack of energy. Both factors within the children (temperament, lifestyle) and external factors (the environment and treatment modalities) affected how children responded to fatigue. These are important insights into a complex phenomenon, and, while varied facets of fatigue are addressed in the PedsQL Multidimensional Fatigue Scale, these perspectives may not be represented in the instrument. This requires further examination.

The results of this study confirm the usefulness of the PedsQL Multidimensional Fatigue Scale for this purpose in Arabic children with cancer. The translated PedsQL Multidimensional Fatigue Scale and its subscales demonstrated excellent internal consistency reliability. Reliability ranged from 0.87 to 0.94 ,) except for sleep/rest fatigue (alpha=0.67). Cronbach alpha score was 0.90 for the total PedsQL Multidimensional Fatigue Scale, which was a little better than in recent studies in children (Varni et al, 2002a; Nascimento et al, 2015).

Moreover, the construct validity of the PedsQL Multidimensional Fatigue was established as it converged well with the validated measure of PedsQL 4.0 Generic Core scale and all subscales. The positive correlation coefficients indicated that higher Fatigue Total Scale and Subscale scores (fewer problems or symptoms) were correlated with higher Generic Core Total Scale Scores (better overall HRQOL). There is evidence from Varni et al (2002a) and Kobayashi et al (2011) to demonstrate a significant positive correlation between the PedsQL Multidimensional Fatigue Scale and the PedsQL 4.0 Generic Core scale and all subscales. This could be considered as additional evidence to suggest that the PedsQL Multidimensional Fatigue Scale (Arabic version) is a valid instrument. 
Instruments used with children and young people in a general population also tend to show worse results for children with cancer. In a Lebanese study, Sabbah et al. (2012) translated the PedsQL Generic Core Scale into Lebanon Arabic for use with 5-12 year-olds. Overall health-related quality of life was poorest for children with cancer, even in a population with intensely problematic habitat and widespread emotional deficit. The importance of producing and testing regionally usable versions of instruments is clear when access to valid data from such populations is achieved.

This study has some limitations. The sample size in this study prevented the application of factor analysis to estimate construct validity. Tabachnick \& Fidell (1996) recommend at least 300 cases for factor analysis. However, Cronbach alpha for the PedsQL Multidimensional Fatigue Scale and its subscales were good, indicating reasonable internal consistency. This study is limited to Arabic children with cancer and is not be applicable to other children. Replication of this study with patients from other Arabic countries would be useful, and a larger study is planned to overcome some of the limitations noted here.

\section{Conclusion}

The PedsQL Multidimensional Fatigue Scale (Arabic version) is an effective, reliable and valid instrument to assess fatigue among Arabic children with cancer. This instrument could help nurses and other health care providers both in clinical practice and in research to apply and evaluate interventions aimed at reduction of children's' fatigue following a diagnosis of cancer. This study should raise awareness in nurses and other health care providers that fatigue symptoms are a potential problem for children with cancer. A similar study in the UK is ongoing, and the results from that study will be 
compared to this one in due course. Early diagnosis using a reliable and valid instrument is considered to be of vital importance. 


\section{References}

(2016). Health-related quality of life and its association with self-esteem and fatigue among children diagnosed with cancer. Journal of Clinical Nursing. 25: 3391-3399, doi: 10.1111/jocn.13467

(2009). Development of a modified instrument to measure anticipatory grieving in Jordanian parents of children diagnosed with cancer: the MM-CGI Childhood Cancer. Cancer Nursing 32(3) 211-219. DOI: 10.1097/NCC.0b013e31819a2ae4

Arabiat, D., Elliott, B., Draper, P. \& Al Jabery. (2011). Cross-cultural validation of the Pediatric Quality of Life Inventory 4.0 (PedsQL) generic core scale into Arabic Language. Scandinavian Journal of Caring Sciences, 25,828-833.

Bakker, R. J., van de Putte, E. M., Kuis, W., \& Sinnema, G. (2009). Risk factors for persistent fatigue with significant school absence in children and adolescents. Pediatrics, 124, 89-95.

Brislin, R.W. (1986). The wording and translation of research instrument. InW. J. Lonner \& J.W. Berry (Eds.), Field methods in cross-cultural research (pp. 137-164). Beverly Hills, CA: Sage Publications.

Campos, M. P. O., Hassan, B. J., Riechelmann, R., \& Del Giglio, A. (2011). Cancer related fatigue: A review. Journal of Brazilian Medical Association, 57, 211-219.

Davies, B., Whitsett, SF., Bruce, A., \& McCarthy, P. (2002). A typology of fatigue in children with cancer. Journal of Pediatric Oncology Nursing 19(1): 12-21. DOI: 10.1053/jpon.2002.30012 
Erickson, J., Beck, S., Dudley, Christian, B., Hollen, P., Albritton, K., Godder, K. (2010). Patterns of fatigue in adolescents receiving chemotherapy. Oncology Nursing Forum, 37:444-455.

Gibson, F., Mulhall, A. B., Richardson, A., Edwards, J. L., Ream, E., \& Sepion, B. J. (2005). A phenomenologic study of fatigue in adolescents receiving treatment for cancer. Oncology Nursing Forum, 32, 651-660.

Gordijn, M. S., Cremers, E. M. P., Kaspers, G. J., \& Gemke, R. J. (2011). Fatigue in children: Reliability and validity of the Dutch PedsQL Multidimensional Fatigue Scale. Quality of Life Research, 20, 1103-1108.

Hinds, P. S., Hockenberry, M., Tong, X., Rai, S. N., Gattuso, J. S., McCarthy, K., ... Srivastava, D. K. (2007). Validity and reliability of a new instrument to measure cancer-related fatigue in adolescents. Journal of Pain and Symptom Management, 34(6), 607. doi.org/10.1016/j.jpainsymman.2007.01.009

Hockenberry-Eaton, M. J., Hinds, P. S., Barrera, P., Bryant, R., Adams-McNeill, J., Hooke, C., . . . Manteuffel, B. (2003). Three instruments to assess fatigue in children with cancer: the child, parent and staff perspectives. Journal of Pain and Symptom Management, 25( 4), 319-28.

Jóhannsdóttir, I. M.R., Hjermstad, M. J., Moum, T., Wesenberg, F., Hjorth, L., Schrøder, H., Mört, S., Jónmundsson, G. and Loge, J. H. (2012). Increased prevalence of chronic fatigue among survivors of childhood cancers: A population-based study. Pediatric Blood \& Cancer 58: 415-420. doi:10.1002/pbc.23111 
Ministry of Health. (2010). Jordan Cancer Registry. The Hashemite Kingdom of Jordan. Ministry of Health.

Kobayashi, K., Okano, Y., \& Hohashi, N. (2011). Reliability and validity of the PedsQL Multidimensional Fatigue Scale in Japan. Quality of Life Research, 20, 1091-1102.

Lai, J. S., Cella, D., Kupst, M. J., Holm, S., Kelly, M. E., Bode, R. K., \& Goldman, S. (2007). Measuring fatigue for children with cancer: Development and validation of the pediatric Functional Assessment of Chronic Illness Therapy-Fatigue (pedsFACIT-F). Journal of Pediatric Hematology/ Oncology, 29(7), 471-9.

McCorkle, R. (1987). The measurement of symptom distress. Seminars in Oncology Nursing 3:248256.

Miyauti-Silva, MC., Lopes-Júnior, LC., Nascimento, LC., \& Lima, RAG. (2016). Fatigue in children and adolescents with cancer from the perspective of health professionals. Revista Latino-Americana de Enfermagem 24:e2784. doi.org/10.1590/1518-8345.1159.2784.

Nascimento, L., Nunes, M., Rocha, E., Bomfim, E., Santos, M., Santos, C., .... Lima, R. (2015). High validity and reliability of the PedsQL Multidimensional Fatigue Scale for Brazilian children with cancer. Journal of Pediatric Oncology Nursing 32(1), 57-64. 
Polit, D. \& Beck, C. (2008). Nursing research: generating and assessing evidence for nursing practice, 8th edn. Philadelphia, PA: Lippincot Williams and Wilkins.

Portenoy, R., Thaler, T., Kornblith, A., Lepore, M., Friedlander-Klar, H., Kiyasu, E.... Scher, H. (1994). The Memorial Symptom Assessment Scale: an instrument for the evaluation of symptom prevalence, characteristics and distress. European Journal of Cancer, 30A: 1326-1336

Sabbah, I., Sabbah, H., Sabbah, S., Akoum, H., Droubi, N., \& Mercier, M. (2012) Measurement properties of the Arabic Lebanon version of the Pediatric Quality of Life Inventory 4.0 Generic Core Scales for young child (5 - 7 years), and child aged 8 - 12 years: quality of life in urban and rural children in Lebanon. Creative Education 3(Special Issue): 959-970. doi 10.4236/ce.2012.326146

Tabachnick, B., and Fidell, L. (2007) Using multivariate statistics (5th Ed) USA: Pearson Education, Inc

Tomlinson, D., Hinds, P., Ethier, M., Ness, K., Zupanec, S., Sung, L. (2013) Psychometric properties of instruments used to measure fatigue in children and adolescents with cancer: A systematic review. Journal of Pain and Symptom Management, 45:83-91.

Varni, J. W., Burwinkle, T. M., Katz, E. R., Meeske, K., \& Dickinson, P. (2002a). The PedsQL in pediatric cancer: Reliability and validity of the Pediatric Quality of Life Inventory Generic Core Scales, Multidimensional Fatigue Scale, and Cancer Module. Cancer, 94, 2090-2106. 
Varni, J.W., Seid, M., Knight, T.S., Uzark, K., \& Szer, I.S. (2002b). The PedsQL 4.0 Generic Core Scales: Sensitivity, responsiveness, and impact on clinical decision-making. Journal of Behavioral Medicine, 25, 175-193.

Varni, J. W., Burwinkle, T. M., \& Szer, I. S. (2004). The PedsQL Multidimensional Fatigue Scale in pediatric rheumatology: Reliability and validity. Journal of Rheumatology, 31, 2494-2500.

Yılmaz, HB., Gerçeker, GÖ., Muslu, GK. (2016). Evaluating the cancer related fatigue by children, mothers, and nurses in Turkish pediatric oncology patients. European Journal of Oncology Nursing 23: 66-71. doi: 10.1016/j.ejon.2016.05.002

Varni, J. W., \& Limbers, C. A. (2008). The PedsQL Multidimensional Fatigue Scale in young adults: Feasibility, reliability and validity in a university student population. Quality of Life Research, 17, 105114. 
Table 1: Item-total scale correlations and item-subscales correlations $(n=70)$

\section{Subscales/item}

number
Item-subscale

correlation
Item-total scales

correlation

\section{General fatigue subscale}

1 feel tired

$0.84 * *$

$0.75 * *$

2 feel physically weak (not

$0.91 * *$

0. $77 * *$ strong)

3 feel too tired to do things that

$0.82 * *$

0. $74 * *$

I like to do

4 feel too tired to spend time

$0.85 * *$

0. $71 * *$

with my friends

5 have trouble finishing things

$0.90 * *$

0. $75 * *$

6 have trouble starting things

$0.90 * *$

0. $77 * *$

\section{Sleep/Rest fatigue subscale}

1 sleep a lot

$0.68 * *$

$0.41 *$

2 It is hard to sleep through the night

$0.43 * *$

0.39*

3 feel tired when waking up in the morning

$0.48 * *$

$0.53 * *$

4 rest a lot

$0.61 * *$

$0.48 * *$

5 take a lot of naps

$0.71 * *$

$0.43 * *$

6 spend a lot of time in bed

$0.75 * *$

$0.40 * *$

\section{Cognitive fatigue subscale}

1 It is hard for me to keep my

$0.53 * *$

$0.53 * *$

attention on things

2 It is hard for me to remember

$0.90 * *$

$0.65 * *$

what people tell me 
3 It is hard for me to remember

$0.80 * *$

$0.59 * *$

what I just heard

4 It is hard for me to think

$0.81 * *$

$0.70 * *$

quickly

5 I have trouble remembering

$0.89 * *$

$0.61 * *$

what I was just thinking

6 I have trouble remembering

$0.77 * *$

$0.60 * *$

more than one thing at a time 
Table 2: PedsQL Multidimensional Fatigue Scale Internal Consistency Reliability by Age and Summary Score/Subscales

\begin{tabular}{ccccc}
\hline \multicolumn{1}{c}{ Scale } & \multicolumn{3}{c}{ Age Group (yrs) } & Total sample \\
& Young child (5-7) & Child (8-12) & $(\mathbf{1 3 - 1 8 )}$ & \\
& & & & \\
\hline Total fatigue & 0.84 & 0.87 & 0.90 & $\mathbf{0 . 9 0}$ \\
\hline General fatigue & 0.95 & 0.94 & 0.92 & $\mathbf{0 . 9 4}$ \\
\hline Sleep/rest fatigue & 0.60 & 0.70 & 0.69 & $\mathbf{0 . 6 7}$ \\
\hline Cognitive fatigue & 0.86 & 0.86 & 0.90 & $\mathbf{0 . 8 7}$ \\
\hline
\end{tabular}


Table 3: Pearson Correlation Coefficients between Generic PedsQL Scales and PedsQL Multidimensional Fatigue Scale

\begin{tabular}{|c|c|c|c|c|}
\hline Scale & Total Fatigue & General Fatigue & $\begin{array}{c}\text { Sleep/rest } \\
\text { Fatigue }\end{array}$ & $\begin{array}{c}\text { Cognitive } \\
\text { Fatigue }\end{array}$ \\
\hline $\begin{array}{l}\text { The PedsQL } \\
\text { Generic } \\
\text { 4.0Total score }\end{array}$ & $.690 * *$ & $.654 * *$ & $.524 * *$ & $.441 * *$ \\
\hline $\begin{array}{l}\text { Physical } \\
\text { functioning }\end{array}$ & $.563 * *$ & $.487 * *$ & $.493^{* *}$ & $.353 * *$ \\
\hline $\begin{array}{l}\text { Emotional } \\
\text { functioning }\end{array}$ & $.444 * *$ & $.524 * *$ & $.246^{* *}$ & $.242 *$ \\
\hline $\begin{array}{l}\text { Social } \\
\text { functioning }\end{array}$ & $.364 * *$ & $.332 * *$ & $.272 *$ & $.255^{*}$ \\
\hline School & $.596^{* *}$ & $.497 * *$ & $.516^{* *}$ & $.398 * *$ \\
\hline
\end{tabular}

Functioning

$\begin{array}{lllll}\text { Psychosocial } & .615^{* *} & .602 * * & .453 * * & .384 * *\end{array}$

Health

Summary

** Correlation is significant at the 0.01 level (2-tailed).

* Correlation is significant at the 0.05 level (2-tailed). 
